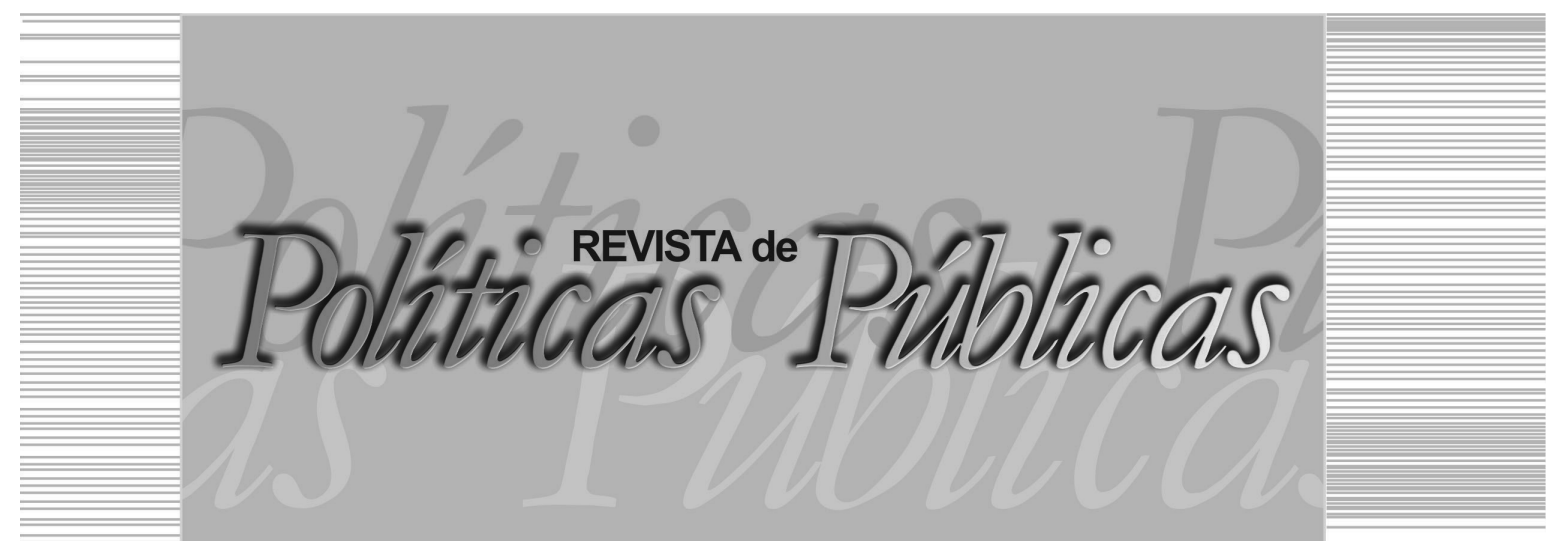

\title{
INSTRUMENTOS DE GESTAO DA ASSISTÊNCIA \\ SOCIAL: uma análise da realidade dos municípios brasileiros
}

\author{
Janaildo Soares de Sousa ${ }^{l}$ \\ Universidade Federal do Ceará (UFC) \\ Donária Linhares Minervino ${ }^{2}$ \\ Secretaria de Assistência Social de São Bento - PB \\ Patrícia Verônica Pinheiro Sales Lima \\ Universidade Federal do Ceará (UFC) \\ Leonardo Andrade Rocha ${ }^{4}$ \\ Universidade Federal Rural do Semiárido (UFERSA) \\ Márcio Regys Rabelo de Oliveira ${ }^{5}$ \\ Universidade Federal do Ceará (UFC)
}

\footnotetext{
Economista, Doutorando em Desenvolvimento e Meio Ambiente pela Universidade Federal do Ceará (UFC). E-mail: janaildo18@hotmail.com

2 Assistente Social, Especialista em Gestão e Avaliação de Políticas Públicas e Sociais pela Faculdades Integradas de Patos (FIP), Assistente Social na Secretaria de Assistência Social de São Bento - PB. E-mail: donaria linhares@hotmail.com / Secretaria de Assistência Social de São Bento - PB: Praça Pedro Eulampio da Silva, 52, Centro, São Bento - PB. CEP: 58.865-000

3 Engenheira Agrônoma, Doutora em Economia Aplicada pela Escola Superior de Agricultura Luiz de Queiroz (ESALQ) da Universidade de São Paulo (USP), Bolsista Produtividade - CNPq, Professora da UFC. E-mail: pvpslima@gmail.com

4 Economista, Doutor em Desenvolvimento Econômico - Instituto de Economia (IE) da Universidade Estadual de Campinas (UNICAMP/SP), Professor da Universidade Federal Rural do Semiárido (UFERSA). E-mail: leonardoandrocha@yahoo.com.br / Universidade Federal Rural do Semiárido - UFERSA: Av. Francisco Mota, 572 - Bairro Costa e Silva, Mossoró-RN. CEP: 59625-900

5 Engenheiro Agronônomo, Mestrando em Engenharia Agrícola pela UFC. E-mail: marcioregys@yahoo.com.br / Universidade Federal do Ceará - UFC: Av. da Universidade, 2853 - Benfica, Fortaleza - CE. CEP: 60020-181
} 
Janaildo Soares de Sousa $\mid$ Donária Linhares Minervino $\mid$ Patrícia Verônica Pinheiro Sales Lima Leonardo Andrade Rocha $\mid$ Márcio Regys Rabelo de Oliveira

\title{
Resumo
}

O objetivo deste estudo é analisar os níveis de adoção dos instrumentos básicos de gestão municipal de assistência social nos municípios brasileiros. Para tanto, utiliza dados da pesquisa Perfil dos Municípios Brasileiros - 2013 publicada pelo IBGE. Como estratégia empírica adota a criação do Índice de Gestão Municipal de Assistência Social para os municípios brasileiros. Os resultados mostram que, embora existam municípios que implementem todos os instrumentos de gestão municipal de assistência social avaliados, em média, o nível de implementação de instrumentos é baixo. $\mathrm{O}$ instrumento menos frequente nas administrações municipais é a lei municipal de regulamentação do SUAS. Conclui que essa deficiência é preocupante e pode reduzir os impactos da política de assistência social, dado que cabe ao SUAS a operacionalização de tais políticas, e a falta de regulamentação pode ser um risco ao direito do cidadão e a negação de uma assistência social assegurada por lei.

Palavras-chave: Instrumentos, mecanismos, gestão municipal, assistência social.

\section{INSTRUMENTS OF SOCIAL ASSISTANCE \\ MANAGEMENT: an analysis of the reality of Brazilian municipalities}

\begin{abstract}
This article aims to analyze the adoption of instruments of social assistance management in Brazilian municipalities. For this purpose it was used the data contained in the Profile of Brazilian Municipalities - 2013, published by IBGE. The empirical strategy adopted was the creation of the social assistance management Index for the Brazilian municipalities. The results showed that, although there are municipalities that implement all instruments of social assistance management evaluated, the average level of instrument implementation is low. The least frequent instrument in municipal administrations is the municipal regulation law of SUAS. Concludes that this deficiency is worrisome and can reduce the impacts of welfare policies since SUAS is responsible for the operationalization of such policies and the lack of regulation can be a risk to the right of the citizen and the denial of a social assistance guaranteed in law. Key words: Instruments, mechanisms, municipal management, social assistan-
\end{abstract} ce.

\section{INTRODUÇÃO}

A descentralização governamental promovida pela Constituição Federal (CF) de 1988 é causa de debates polarizados que ainda não foram consensuados. De um lado, alguns defendem tratar-se de 
INSTRUMENTOS DE GESTÃO DA ASSISTÊNCIA SOCIAL:

uma análise da realidade dos municípios brasileiros

um processo democrático que fortalece os diferentes entes federados (União, Estados, Distrito Federal e Municípios) e favorece a inovação na gestão pública. De outro, há aqueles que se colocam contrários, pois acreditam que os estados e municípios são locais onde prevalecem o clientelismo e a ineficiência administrativa. (MELO, 1997). Apesar das divergências, é crível que a descentralização tem estimulado avanços na criação de instrumentos de gestão capazes de auxiliar na implementação de políticas públicas setoriais.

No âmbito das políticas de assistência social a descentralização político administrativa está prevista no artigo $204^{\circ}$ da CF/1988, o qual estabelece que cabe às esferas estadual e municipal, bem como a entidades beneficentes e de assistência social, a coordenação e execução das ações governamentais na temática. (BRASIL, 1988). Assim, o município tem autonomia institucional para gerir as políticas públicas de assistência social. Tal autonomia requer a adoção de múltiplos instrumentos de gestão, pois se trata de mecanismos que possibilitam uma maior articulação das demandas da população, além de contribuir no melhor uso dos recursos, tendo em vista a sua função fiscalizadora. (CAMPOS, 2004).

Os instrumentos de gestão pública municipal estão previstos em legislação e são considerados elementos essenciais para realizar o controle social de políticas públicas setoriais, garantindo os princípios da participação da sociedade nos processos de decisão em todas as etapas das políticas públicas como emana a Constituição. (NEVES; SANTOS; SILVA, 2012). Na esfera municipal nota-se o baixo interesse governamental na implementação de tais instrumentos. Essa análise foi confirmada nos estudos de Carvalho, Lima e Sousa (2013), Lima e outros (2014) e Sousa, Lima e Khan (2015) nos setores responsáveis pelas políticas de saneamento básico, segurança pública e direitos humanos, respectivamente. Contudo, não existem estudos similares quanto à assistência social.

O interesse pela análise da implementação de instrumentos de gestão da assistência social na esfera municipal torna-se relevante, pois o município é o local onde os serviços públicos são prestados diretamente ao cidadão. (SILVA, 2003). O município é o ente federado mais próximo da realidade e dos problemas da população em geral e com maior autonomia para mobilizar o público alvo dos serviços socioassistenciais. Além disso, as bases constitucionais que envolvem a assistência social no Brasil tem passado por processos 
de regulamentação que culminaram na elaboração de instrumentos cuja implementação ainda não foi empiricamente analisada em escala municipal. É o caso, por exemplo, da Lei Orgânica de Assistência Social (LOAS, 1993) e do Sistema Único de Assistência Social (SUAS, 2005). Por fim, compreende-se que instrumentos e mecanismos de gestão são "[...] partes constitutivas da dinâmica organizacional de todas e quaisquer instituições de natureza púbica ou privada." (KLEBA; COMERLATTO; FROZZA, 2016, p. 1062).

Acredita-se que nos municípios onde há uma maior quantidade de instrumentos de gestão municipal de assistência social, há também uma maior possibilidade de efetividade das políticas assistenciais em âmbito local. Nessa perspectiva, o presente artigo tem por objetivo analisar os níveis de implementação dos instrumentos básicos de gestão municipal de assistência social nos municípios brasileiros, bem como, identificar quais instrumentos são mais ou menos frequentemente implementados.

\section{INSTRUMENTOS DE GESTÃO PÚBLICA MUNICIPAL DE ASSISTÊNCIA SOCIAL NO BRASIL}

A CF/1988, no artigo $18^{\circ}$, reconheceu os Municípios como entes federativos: "A organização político administrativa da República Federativa do Brasil compreende a União, os Estados, o Distrito Federal e os Municípios, todos autônomos [...]”. (BRASIL, 1988). Desde então houve um crescente estímulo a um processo de descentralização caracterizado pela transferência e compartilhamento de responsabilidades, poderes e recursos entre as esferas de governo do Brasil, com o objetivo de realizar uma gestão pública mais eficiente, seja na fiscalização ou implementação de ações. Nessa configuração, os municípios passaram a assumir uma série de competências ${ }^{1}$, inclusive no que diz respeito à assistência social. Apesar de a carta magna ter assumido que a assistência social é um direito de todos e dever do Estado, as competências da União, Estados e Municípios nessa temática não estão claramente discriminadas².

O artigo $204^{\circ}$ da $\mathrm{CF} / 1988$ prevê a descentralização político administrativa e a participação da sociedade nas discussões sobre assistência social, mas pode-se afirmar que a organização da assistência social no Brasil encontra-se regulamentada na Lei n. 8.742, promulgada em 7 de dezembro de 1993 e alterada pela Lei $\mathrm{n}^{\circ} 12.435$, de 6 de julho de 2011, conhecida como LOAS. A Lei Orgânica é a 
INSTRUMENTOS DE GESTÃO DA ASSISTÊNCIA SOCIAL:

uma análise da realidade dos municípios brasileiros

principal competência legislativa dos municípios. Como estabelece o artigo $29^{\circ}$ da CF, é a partir dela que o município deve nortear a sua organização. No caso da LOAS, trata-se de um instrumento de gestão municipal a partir do qual o município deve organizar a sua política de assistência social (GARCIA, 2011), incluindo benefícios sociais, programas, formas de financiamento e origem dos recursos a serem aplicados na área social. Dessa forma, busca regulamentar as conquistas sociais no âmbito assistencial. (WANDERLEY, 2015). Segundo a referida Lei, os municípios têm autonomia para fixar suas respectivas Políticas de Assistência Social.

A CF/1988 e a LOAS trouxeram novas perspectivas para a assistência social no Brasil e "[...] abriram espaço iniciando um processo que tem como perspectiva torná-la visível como política pública e direito dos que dela necessita." (YAZBEK, 2012, p. 303). Em continuidade à evolução da política de assistência social no país, destacam-se: i) a aprovação da Política Nacional de Assistência Social (PNAS, 2004), ii) a Norma Operacional Básica (NOB/2005) que instituiu o SUAS (2005) e iii) a Lei 12.435/2011, que alterou a LOAS de 1993, incluindo o SUAS, as unidades de referência, serviços e programas socioassistenciais na legislação nacional. (QUINONERO et al., 2013).

Atualmente, a construção e a implementação do SUAS vêm se caracterizando como uma das prioridades para o avanço da Política de Assistência Social no país (YAZBEK, 2012), haja vista tratar-se do modelo de gestão para a operacionalização das ações assistenciais nos âmbitos federal, estadual e municipal. O SUAS é o responsável pela organização dos serviços assistenciais e tem como objetivos principais o enfrentamento da situação de pobreza e extrema pobreza, a promoção da proteção social ${ }^{3}$, o fortalecimento dos vínculos familiares e comunitários, bem como a participação dos usuários. (BRASIL, 2005). Para tanto, propõe a descentralização de serviços e a criação de instrumentos de gestão, de modo a inserir a população na elaboração da agenda de demandas sociais. (MARQUES; SANTOS, 2016). De acordo com a NOB/2005 os instrumentos de gestão são ferramentas de planejamento técnico e financeiro do SUAS, em nível federal, estadual ou municipal.

No que se refere à gestão pública municipal, os municípios contam com diferentes elementos para que alcancem a democratização das políticas de assistência social, promovendo a proteção, 
Janaildo Soares de Sousa | Donária Linhares Minervino | Patrícia Verônica Pinheiro Sales Lima Leonardo Andrade Rocha $\mid$ Márcio Regys Rabelo de Oliveira

inclusão e defesa dos direitos. Além da LOAS e do SUAS, a atual legislação prevê a existência de conselhos, planos e fundos municipais de assistência social. Esses instrumentos fazem parte de um modelo de gestão pública que busca articular planejamento e orçamento de forma descentralizada, porém com a manutenção da integração entre união, estados e municípios e o envolvimento da população. (BULLA; LEAL, 2004). Os conselhos, planos e fundos municipais de assistência social integram a gestão pública do SUAS no município. (BRASIL, 2008).

De acordo com o art. $16^{\circ}$ da LOAS, os conselhos municipais de assistência social são instâncias deliberitativas, de caráter permanente e composição paritária entre governo e sociedade civil. (BRASIL, 1993). "Os Conselhos são mecanismos de cooperação na gestão pública, ora como canais democráticos ou como instrumentos de participação pública, averiguando se as ações executadas pelo Estado se pautam em bases legais e atendem ao interesse coletivo." (KLEBA; COMERLATTO; FROZA, 2015, p. 1063).

O Plano Municipal de Assistência Social é um dos principais instrumentos da gestão municipal da assistência social, pois:

É um instrumento de planejamento estratégico que organiza regula
e norteia a execução da PNAS/2004 na perspectiva do SUAS. Sua
elaboração é de responsabilidade do órgão gestor da Política que
o submete à aprovação do Conselho de Assistência Social reafir-
mando o princípio democrático e participativo. (BRASIL, 2004,
p. 119).

O Fundo Municipal de Assistência Social tem como objetivo disponibilizar meios para financiar os serviços, programas e projetos assistenciais do município. Cabe aos gestores municipais, de forma autônoma, a aplicação dos recursos. Porém é necessário que as prioridades de aplicação tenham sido definidas no Plano Municipal de Assistência Social aprovado pelo Conselho Municipal de Assistência Social. (SILVA, 2004).

Pelo exposto, a descentralização e a participação popular são elementos centrais da política de assistência social no Brasil pós-constituição de 1988, a qual é operacionalizada via SUAS. No entanto, ainda existem desafios na consolidação dos princípios e diretrizes propostos pelo SUAS. (BOVOLENTA, 2011). No âmbito municipal esses desafios envolvem uma maior articulação com governos federal e estadual no planejamento, coordenação e execu- 
INSTRUMENTOS DE GESTÃO DA ASSISTÊNCIA SOCIAL:

uma análise da realidade dos municípios brasileiros

ção das ações assistenciais, o que perpassa pela implementação dos instrumentos descritos.

\section{PROCEDIMENTOS METODOLÓGICOS}

$\mathrm{O}$ artigo adotou como unidade de observação os $5.570 \mathrm{mu}-$ nicípios brasileiros ${ }^{4}$. No entanto, devido ao grande número de municípios, os resultados obtidos são discutidos em escala estadual. Os dados utilizados foram extraídos da pesquisa Perfil dos Municípios Brasileiros - Assistência Social, 2013 - publicada pelo Instituto Brasileiro de Geografia e Estatística (IBGE, 2013). A pesquisa traz as informações mais recentes sobre os principais instrumentos de gestão municipal da Assistência Social. Cada município do país tem a possibilidade de implementar qualquer um dos instrumentos mencionados, sendo que a maioria desses instrumentos não requer obrigatoriedade de adoção. Assim, alguns municípios implementam mais e outros menos.

Acredita-se que a adoção dos instrumentos básicos de gestão da Assistência Social pode contribuir para a efetividade das políticas públicas nessa área, o que permite assumir uma relação de causalidade na qual maior nível de implementação de instrumentos de gestão possibilita melhores resultados no que diz respeito ao acesso da população aos serviços de assistência social. Nessa perspectiva, buscou-se mensurar o nível de implementação de instrumentos de gestão municipal da assistência social a partir da agregação de um conjunto de 10 indicadores selecionados do banco de dados da pesquisa, conforme exposto no Quadro 1:

Quadro 1 - Indicadores componentes do Índice de Gestão Municipal de Assistência Social - IGMAS.

\begin{tabular}{|l|}
\hline \multicolumn{1}{|c|}{ Indicadores } \\
\hline 1 - Existência de órgão gestor da assistência social \\
\hline 2 - Realização de cursos para formação de pessoal ocupado na área de assistência social \\
\hline 3 - Existência de Lei Orgânica trata de assistência social - LOAS \\
\hline 4 - Existência de Lei Municipal de regulamentação do SUAS \\
\hline 5 - Existência de Plano Municipal de Assistência Social \\
\hline 6 - Existência de Conselho Municipal de Assistência Social \\
\hline 7 - Existência de Fundo Municipal de Assistência Social \\
\hline 8 - Existência de Fundo Municipal da Criança e do Adolescente \\
\hline 9 - Existência de Fundo Municipal do Idoso \\
\hline 10 - Existência de Fundo Municipal da Pessoa com Deficiência \\
\hline
\end{tabular}

Fonte: Elaborado pelos autores a partir da Pesquisa Perfil dos Municípios Brasileiros - Assistência Social: INSTITUTO BRASILEIRO DE GEOGRAFA E ESTATÍSTICA. Perfil dos municípios brasileiros Assistência Social 2013. Rio de Janeiro, 2013. Pesquisa de informações básicas municipais. 
Janaildo Soares de Sousa | Donária Linhares Minervino | Patrícia Verônica Pinheiro Sales Lima Leonardo Andrade Rocha $\mid$ Márcio Regys Rabelo de Oliveira

A agregação dos indicadores foi realizada conforme proposto por Sousa, Lima e Khan (2015) e resultou no Índice de Gestão Municipal de Assistência Social (IGMAS). Os indicadores foram quantificados por meio da atribuição de escores, e esta ocorreu da seguinte forma: foi atribuído escore 1 (um) na existência do instrumento no município e 0 (zero).na ausência deste. $\mathrm{O}$ procedimento matemático para a agregação consistiu na aplicação da equação:

$$
I_{\text {IGMAS }}=\sum_{i=1}^{p} \frac{E_{i j}}{E \max i}
$$

Sendo:

IGMAS = Índice de Gestão Municipal de Assistência Social observado no j-ésimo município

$\mathrm{E}_{\mathrm{ij}}=$ escore do i-ésimo indicador obtido pelo j-ésimo município

$\mathrm{E}_{\text {maxi }}=$ escore máximo do i-ésimo indicador

$\mathrm{i}=1, \ldots \ldots . . ., p$, número de indicadores $(\mathrm{p}=10)$

$\mathrm{j}=1, \ldots \ldots \ldots, \mathrm{n}$, número de municípios $(\mathrm{n}=5.570)$

O referido IGMAS permite demonstrar o nível de implementação dos mecanismos em análise em nível municipal, variando de 0 (zero) a 1 (um). Quanto mais próximo 1, maior é o nível de adoção dos indicadores nos respectivos municípios. Ademais, quando multiplicado por 100, o IGMAS pode ser interpretado como o percentual de implementação dos instrumentos municipais da gestão de assistência social no município.

Como mencionado, o IGMAS foi calculado para cada um dos municípios do Brasil. Porém, optou-se por agregar as referidas informações por unidade da federação. Desse modo, o índice de cada unidade federativa refere-se à média aritmética do IGMAS dos municípios que a compõem.

Em seguida foi realizada uma análise de agrupamento para classificação das unidades federativas em três grupos. Para isso, foi empregada como método de agrupamento a técnica k-médias, como sugerido por Hair Jr. e outros (2005). Os níveis de implementação de instrumentos de gestão definidos para cada classe foram:

$0,59 \leq$ IGMAS $\leq 0,62$ - Municípios com menores níveis de adoção de mecanismos de gestão municipal de assistência social; 
INSTRUMENTOS DE GESTÃO DA ASSISTÊNCIA SOCIAL:

uma análise da realidade dos municípios brasileiros

$0,63 \leq \mathrm{IGMAS} \leq 0,67$-Municípios com níveis intermediários de adoção dos mecanismos de gestão municipal de assistência social;

$0,70 \leq \mathrm{IGMAS} \leq 0,71-$ Municípios com maiores níveis de adoção de mecanismos de gestão municipal de assistência social.

Por fim, para uma melhor visualização da distribuição espacial do IGMAS no território brasileiro, foi construído um mapa temático com o uso do software ArcGis. V10.

\section{RESULTADOS E DISCUSSÃO}

A política de assistência social no Brasil foi implementada para reduzir os problemas sociais instalados ao longo dos anos e, em especial, na última década pelo agrave da pobreza e exclusão social promovida pelas mudanças de caráter institucional, econômico, tecnológico, política e cultural. (PERES; ALVES, 2009). A partir da divisão de competências regulamentada na NOB/2005, coube aos municípios um novo protagonismo na gestão dos serviços, programas, projetos e benefícios assistenciais, o que gerou a demanda por instrumentos capazes de operacionalizar as diretrizes da assistência social no âmbito local.

A Tabela 1 mostra o IGMAS que expressa, em termos médios, o grau de adoção dos municípios em relação aos dez mecanismos de gestão de assistência social avaliados. Mais de duas décadas após a aprovação da LOAS e com uma década de SUAS, os resultados do estudo apontam que a implementação de instrumentos de gestão que poderiam auxiliar o município na gestão da assistência municipal é relativamente baixa.

Tabela 1 - Estatísticas descritivas do IGMAS, por unidade federativa - Ano base 2013

\begin{tabular}{c|c|c|cc|cc}
\hline $\begin{array}{c}\text { Unidade } \\
\text { Federativa }\end{array}$ & $\begin{array}{c}\text { Total de } \\
\text { Municípios }\end{array}$ & $\begin{array}{c}\text { Índice Médio } \\
\text { da Gestão } \\
\text { Municipal de } \\
\text { Assistência } \\
\text { Social - } \\
\text { IGMAS }\end{array}$ & Mediana & Mínimo & Máximo & $\begin{array}{c}\text { Coeficiente } \\
\text { de Variação } \\
(\%)\end{array}$ \\
\hline Acre & 22 & 0,60 & 0,60 & 0,40 & 0,70 & 11,9 \\
\hline Alagoas & 102 & 0,64 & 0,60 & 0,50 & 1,00 & 14,3 \\
\hline Amazonas & 62 & 0,61 & 0,60 & 0,40 & 1,00 & 19,6 \\
\hline Amapá & 16 & 0,63 & 0,60 & 0,50 & 0,80 & 13,7 \\
\hline
\end{tabular}


Janaildo Soares de Sousa | Donária Linhares Minervino | Patrícia Verônica Pinheiro Sales Lima Leonardo Andrade Rocha $\mid$ Márcio Regys Rabelo de Oliveira

\begin{tabular}{|c|c|c|c|c|c|c|}
\hline $\begin{array}{l}\text { Unidade } \\
\text { Federativa }\end{array}$ & $\begin{array}{c}\text { Total de } \\
\text { Municípios }\end{array}$ & $\begin{array}{c}\text { Índice Médio } \\
\text { da Gestão } \\
\text { Municipal de } \\
\text { Assistência } \\
\text { Social - } \\
\text { IGMAS }\end{array}$ & Mediana & Mínimo & Máximo & $\begin{array}{c}\text { Coeficiente } \\
\text { de Variação } \\
(\%)\end{array}$ \\
\hline Bahia & 417 & 0,63 & 0,60 & 0,30 & 1,00 & 18,9 \\
\hline Ceará & 184 & 0,63 & 0,60 & 0,40 & 0,90 & 15,4 \\
\hline $\begin{array}{l}\text { Distrito } \\
\text { Federal }\end{array}$ & 1 & 0,70 & - & - & - & - \\
\hline $\begin{array}{c}\text { Espírito } \\
\text { Santo }\end{array}$ & 78 & 0,62 & 0,60 & 0,30 & 0,90 & 18,4 \\
\hline Goiás & 246 & 0,66 & 0,60 & 0,40 & 1,00 & 18,3 \\
\hline Maranhão & 217 & 0,64 & 0,60 & 0,00 & 1,00 & 19,4 \\
\hline $\begin{array}{l}\text { Minas } \\
\text { Gerais }\end{array}$ & 853 & 0,64 & 0,60 & 0,30 & 1,00 & 18,5 \\
\hline $\begin{array}{c}\text { Mato Grosso } \\
\text { do Sul }\end{array}$ & 79 & 0,70 & 0,70 & 0,50 & 0,90 & 11,9 \\
\hline Mato Grosso & 141 & 0,66 & 0,60 & 0,20 & 1,00 & 19,9 \\
\hline Pará & 144 & 0,63 & 0,60 & 0,10 & 1,00 & 17,9 \\
\hline Paraíba & 223 & 0,64 & 0,60 & 0,30 & 1,00 & 19,2 \\
\hline Pernambuco & 185 & 0,64 & 0,60 & 0,40 & 0,90 & 18,8 \\
\hline Piauí & 224 & 0,64 & 0,60 & 0,40 & 1,00 & 16,9 \\
\hline Paraná & 399 & 0,70 & 0,70 & 0,30 & 1,00 & 16,4 \\
\hline $\begin{array}{l}\text { Rio de } \\
\text { Janeiro }\end{array}$ & 92 & 0,60 & 0,60 & 0,40 & 0,80 & 16,3 \\
\hline $\begin{array}{l}\text { Rio Grande } \\
\text { do Norte }\end{array}$ & 167 & 0,59 & 0,60 & 0,30 & 0,90 & 18,5 \\
\hline Rondônia & 52 & 0,65 & 0,60 & 0,40 & 0,90 & 21,5 \\
\hline Roraima & 15 & 0,65 & 0,60 & 0,50 & 0,90 & 17,2 \\
\hline $\begin{array}{c}\text { Rio Grande } \\
\text { do Sul }\end{array}$ & 497 & 0,60 & 0,60 & 0,00 & 1,00 & 22,1 \\
\hline $\begin{array}{c}\text { Santa } \\
\text { Catarina }\end{array}$ & 295 & 0,62 & 0,60 & 0,00 & 1,00 & 17,9 \\
\hline Sergipe & 75 & 0,63 & 0,60 & 0,30 & 0,90 & 20,0 \\
\hline São Paulo & 645 & 0,65 & 0,60 & 0,40 & 1,00 & 17,5 \\
\hline Tocantins & 139 & 0,61 & 0,60 & 0,40 & 0,90 & 15,9 \\
\hline Brasil & 5.570 & 0,64 & 0,60 & 0,00 & 1,00 & 18,7 \\
\hline
\end{tabular}

Fonte: Elaborada pelos autores a partir Pesquisa Perfil dos Municípios Brasileiros - Assistência Social, 2013 (INSTITUTO BRASILEIRO DE GEOGRAFA E ESTATÍSTICA, 2013).

Observando-se o valor da mediana, nota-se que $50 \%$ dos municípios de 24 das 27 unidades federativas implementaram no máximo 6 dos 10 instrumentos avaliados em 2013 (Mediana do IGMAS $=0,60$ ). Não foi observado o emprego de instrumentos em dois municípios (Mato Queimado no RS e Brejo da Areia no MA). Por outro lado, 37 municípios alcançaram índice 1 (apenas 0,7\% 
INSTRUMENTOS DE GESTÃO DA ASSISTÊNCIA SOCIAL:

uma análise da realidade dos municípios brasileiros

dos municípios brasileiros), o que equivale à implementação dos 10 instrumentos avaliados 5 .

Observa-se que os estados do Mato Grosso do Sul e do Paraná, seguidos do Distrito Federal, lideram na implementação dos mecanismos em análise, pois adotam, em média, $70 \%$ dos instrumentos de gestão (IGMAS igual 0,70). No entanto, o caso mais extremo é o dos municípios pertencentes ao estado do Rio Grande do Norte, o que demonstra uma fragilidade do Estado na gestão social (IGMAS igual a 0,59$)$.

A relativamente baixa heterogeneidade entre os municípios, captada pelos coeficientes de variação inferiores a $25 \%$, ficou ainda mais perceptível após a distribuição das unidades federativas nas três classes pré-definidas. Como se observa na Figura 1 a maior parte das unidades federativas posicionou-se na mesma classe, com nível intermediário de implementação de instrumentos de gestão da assistência social. Mato Grosso do Sul, Paraná e Distrito Federal foram classificados como similares e incluídos no grupo de nível mais elevado, enquanto Acre, Amazonas, Rio de Janeiro, Rio Grande do Norte, Rio Grande do Sul, Santa Catarina e Tocantins foram reunidos na classe com menores IGMAS.

Figura 1 - Distribuição do IGMAS nos estados brasileiros por meio de cluster

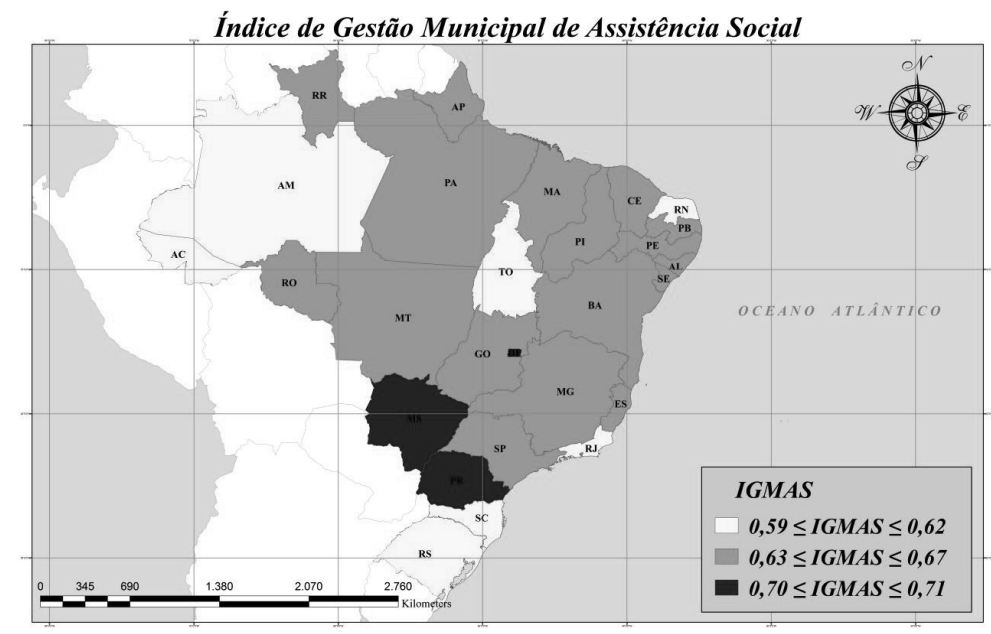

Fonte: Elaborada pelos autores a partir Pesquisa Perfil dos Municípios Brasileiros - Assistência Social, 2013 (INSTITUTO BRASILEIRO DE GEOGRAFA E ESTATÍSTICA, 2013). 
Janaildo Soares de Sousa | Donária Linhares Minervino | Patrícia Verônica Pinheiro Sales Lima Leonardo Andrade Rocha $\mid$ Márcio Regys Rabelo de Oliveira

Segundo Kleba, Comerlatto e Frozza (2015) a existência dos instrumentos de gestão de assistência social nos municípios brasileiros fortalece o poder local para que execute as suas ações, bem como a sociedade civil para que participe de todo o processo decisório das políticas públicas. Os instrumentos ainda servem para que se tenha um equilíbrio de defesa entre os interesses privados e corporativos. (ALMEIDA; TATAGIBA, 2012). Mas, não se pode deixar de mencionar que se trata de uma obediência ao que está previsto na LOAS e de um compromisso que os municípios, enquanto entes federativos, devem assumir para a melhor condução das políticas de assistência social. Embora cada ente federativo tenha suas especificidades, é preciso adotar mecanismos básicos como os descritos, para que se tenha uma gestão eficiente da assistência social e de todas as demais áreas. Sabe-se que não é a simples adoção dos mecanismos que irá mudar a realidade do quadro social, mas a existência destes pode ser o ponto de partida para o alcance e melhoria da gestão pública de assistência social na esfera municipal.

Ollaik e Medeiros (2011) ressaltam que é de suma importância que os entes federados façam uso dos instrumentos de gestão, pois eles produzem efeitos específicos, além disso, dão estrutura às políticas públicas e influenciam nas ações, metas e recursos a serem utilizados pelo gestor. O IGMAS mostrou que a implementação de instrumentos de gestão da assistência social ainda é tímida entre os municípios, o que corrobora o pensamento de Vazquez e outros (2005) quanto à necessidade de uma maior efetivação e uso dos mecanismos institucionais de participação e democratização da gestão pública e do controle social. A título de contribuição adicional do artigo, é possível identificar, na Tabela 2, quais as lacunas das unidades federativas e do Brasil quanto aos instrumentos analisados. A fim de sintetizar a análise, serão descritos os valores referentes ao Brasil (última linha). 
INSTRUMENTOS DE GESTÃO DA ASSISTÊNCIA SOCIAL:

uma análise da realidade dos municípios brasileiros

Tabela 2 - Proporção de municípios com implementação de instrumentos de gestão municipal de Assistência Social, por unidade federativa - Ano 2013

\begin{tabular}{|c|c|c|c|c|c|c|c|c|c|c|}
\hline Unidade Federativa & 1 & 2 & 3 & 4 & 5 & 6 & 7 & 8 & 9 & 10 \\
\hline Acre & 95,5 & 90,9 & 100,0 & 13,6 & 68,2 & 100,0 & 100,0 & 36,4 & 0,0 & 0,0 \\
\hline Alagoas & 82,4 & 80,4 & 98,0 & 17,6 & 99,0 & 100,0 & 100,0 & 46,1 & 8,8 & 3,9 \\
\hline Amazonas & 91,9 & 83,9 & 93,5 & 25,8 & 80,6 & 100,0 & 96,8 & 24,2 & 6,5 & 4,8 \\
\hline Amapá & 62,5 & 100,0 & 100,0 & 56,3 & 100,0 & 100,0 & 100,0 & 6,3 & 0,0 & 0,0 \\
\hline Bahia & 88,5 & 72,2 & 93,5 & 18,5 & 92,1 & 100,0 & 99,0 & 47,5 & 11,3 & 5,5 \\
\hline Ceará & 48,9 & 91,3 & 93,5 & 12,5 & 95,7 & 100,0 & 100,0 & 68,5 & 13,0 & 1,6 \\
\hline Distrito Federal & 100,0 & 100,0 & 100,0 & 100,0 & 100,0 & 100,0 & 100,0 & 0,0 & 0,0 & 0,0 \\
\hline Espírito Santo & 74,4 & 65,4 & 93,6 & 11,5 & 88,5 & 100,0 & 100,0 & 75,6 & 10,3 & 1,3 \\
\hline Goiás & 86,6 & 87,4 & 95,9 & 17,5 & 99,2 & 100,0 & 100,0 & 44,3 & 20,3 & 10,6 \\
\hline Maranhão & 90,8 & 77,0 & 89,9 & 29,0 & 93,5 & 99,1 & 98,6 & 37,3 & 13,8 & 6,5 \\
\hline Minas Gerais & 78,0 & 84,4 & 95,5 & 22,5 & 89,3 & 100,0 & 99,5 & 54,9 & 13,5 & 4,2 \\
\hline Mato Grosso do Sul & 88,5 & 98,7 & 100,0 & 14,1 & 100,0 & 101,3 & 101,3 & 87,2 & 14,1 & 3,8 \\
\hline Mato Grosso & 80,1 & 87,9 & 90,8 & 15,6 & 92,9 & 99,3 & 98,6 & 58,9 & 23,4 & 7,8 \\
\hline Pará & 85,3 & 83,2 & 97,9 & 16,1 & 90,2 & 100,0 & 100,7 & 48,3 & 7,0 & 4,2 \\
\hline Paraíba & 84,8 & 87,9 & 99,1 & 22,0 & 95,1 & 100,0 & 96,9 & 28,7 & 20,6 & 6,3 \\
\hline Pernambuco & 81,1 & 84,3 & 95,7 & 17,8 & 88,6 & 100,0 & 99,5 & 56,8 & 16,8 & 4,3 \\
\hline Piauí & 85,3 & 87,9 & 96,4 & 22,8 & 99,1 & 100,0 & 100,0 & 28,1 & 9,8 & 5,8 \\
\hline Paraná & 90,5 & 86,5 & 93,7 & 26,6 & 98,0 & 100,0 & 99,2 & 74,2 & 24,6 & 6,0 \\
\hline Rio de Janeiro & 68,5 & 73,9 & 88,0 & 7,6 & 92,4 & 100,0 & 100,0 & 55,4 & 14,1 & 2,2 \\
\hline Rio Grande do Norte & 53,3 & 80,2 & 95,2 & 14,4 & 89,2 & 100,0 & 100,0 & 35,9 & 14,4 & 3,0 \\
\hline Rondônia & 69,2 & 69,2 & 88,5 & 26,9 & 100,0 & 100,0 & 100,0 & 59,6 & 26,9 & 7,7 \\
\hline Roraima & 60,0 & 100,0 & 100,0 & 26,7 & 93,3 & 100,0 & 100,0 & 40,0 & 20,0 & 13,3 \\
\hline Rio Grande do Sul & 40,7 & 73,2 & 94,4 & 22,0 & 94,6 & 99,6 & 99,4 & 53,6 & 15,1 & 7,7 \\
\hline Santa Catarina & 53,6 & 87,7 & 94,2 & 15,4 & 98,6 & 100,3 & 100,7 & 54,6 & 9,2 & 4,8 \\
\hline Sergipe & 70,7 & 78,7 & 92,0 & 18,7 & 93,3 & 100,0 & 100,0 & 64,0 & 13,3 & 4,0 \\
\hline São Paulo & 80,5 & 87,1 & 94,1 & 12,9 & 97,7 & 100,0 & 100,0 & 54,7 & 22,2 & 4,8 \\
\hline Tocantins & 78,4 & 93,5 & 97,8 & 18,7 & 95,7 & 100,0 & 97,8 & 17,3 & 7,9 & 2,9 \\
\hline Brasil & 定 & $\bar{\infty}$ & $\begin{array}{l}0 \\
\dot{\sigma}\end{array}$ & $\stackrel{m}{9}$ & \&े & 8. & बें & $\frac{m}{i n}$ & 点 & กี \\
\hline
\end{tabular}

Fonte: Elaborada pelos autores a partir Pesquisa Perfil dos Municípios Brasileiros - Assistência Social, 2013 (INSTITUTO BRASILEIRO DE GEOGRAFA E ESTATÍSTICA, 2013).

Nota: 1 - Existência de órgão gestor da assistência social; 2 - Realização de cursos para formação de pessoal ocupado na área de assistência social, 3 - Existência de Lei Orgânica que trata de assistência social; 4 - Existência de Lei Municipal de regulamentação do SUAS; 5 - Existência de Plano Municipal de assistência social; 6 - Existência de Conselho Municipal de Assistência Social; 7 - Existência de Fundo Municipal de Assistência Social; 8 - Existência de Fundo Municipal da Criança e do Adolescente; 9 - Existência de Fundo Municipal do Idoso e 10 - Existência de Fundo

Municipal da Pessoa com Deficiência. 
Janaildo Soares de Sousa | Donária Linhares Minervino | Patrícia Verônica Pinheiro Sales Lima Leonardo Andrade Rocha $\mid$ Márcio Regys Rabelo de Oliveira

Em 2013, 75,4\% dos municípios brasileiros possuíam Secretaria Municipal de Assistência Social. Em alguns municípios, as políticas públicas eram geridas por setores subordinados a outras secretarias. A implementação do SUAS, bem como do Programa Bolsa Família, contribuiu para fortalecer o aparato institucional e organizacional dos municípios, pois delegou responsabilidades às Secretarias Municipais de Assistência Social. (VAITSMAN; ANDRADE; FARIAS, 2009). Tais responsabilidades impuseram a capacitação de recursos humanos, o que explica o elevado percentual de municípios que ofereceram algum tipo de treinamento aos funcionários públicos da área $(83,1 \%)$.

Quanto à existência de Lei Orgânica, que trata de assistência social, ressalta-se que a elevada frequência do instrumento é um indicativo de autonomia municipal para se organizar nas questões assistenciais. No entanto, apenas 19,3\% dos municípios possuem Lei municipal de regulamentação do SUAS. Esse cenário contraria o Conselho Nacional de Assistência Social (CNAS) e os Pactos de Aprimoramento do SUAS nos municípios, que apregoam a adequação das legislações municipais de modo a atualizar ou instituir a lei que dispõe sobre o SUAS no âmbito municipal, segundo a NOB/ SUAS de 2012. Além disso, retrata uma contravenção, dado que o Pacto de Aprimoramento do SUAS deve ser obrigatoriamente seguido pelos estados, municípios e Distrito Federal, pois possui caráter cogente explicitado no inciso II do art. $18^{\circ}$ da LOAS. (BRASIL, 2012).

Os conselhos, planos e fundos municipais são eixos estruturantes da Política Nacional de Assistência Social e do SUAS em âmbito local. De acordo com o artigo $30^{\circ}$, da LOAS, só haverá repasse de recursos financeiros para a assistência social municipal se o município possuir: i) um Conselho Municipal de Assistência Social; ii) um Fundo Municipal de Assistência Social e iii) um Plano de Assistência Social.

O plano municipal de assistência social expressa as aspirações da política municipal de assistência social e é um dos instrumentos de gestão mais frequentes nas cidades brasileiras. Quanto aos conselhos municipais, nota-se que estão presentes na quase totalidade dos municípios brasileiros, mostrando em termos quantitativos, o interesse municipal na implantação da diretriz de participação popular. Como escreve Abramovay (2001) o aumento no número de 
INSTRUMENTOS DE GESTÃO DA ASSISTÊNCIA SOCIAL:

uma análise da realidade dos municípios brasileiros

conselhos municipais é uma importante inovação institucional das polpiticas públicas. No entanto, apesar do potencial transformador desse instrumento, é importante frisar que a participação popular ainda é precária e, muitas vezes, submissa a poderes dominantes. Marques e Santos (2016), ao estudar a participação social no SUAS, concluíram sobre a fragilidade e pouca legitimação dos conselhos de assistência social como espaços de participação da sociedade civil. Por fim, destaca-se a elevada frequência dos fundos de assistência em comparação a outros fundos que também executam o orçamento da assistência social, caso da criança e do adolescente, do idoso e pessoa com deficiência.

É inegável a contribuição da adoção e uso de mecanismos de gestão, porém, alguns estudos relatam que a grande maioria desses instrumentos não tem alcançado se instituir como instância de diálogo. (RONCONI; DEBETIR; MATTIA, 2011). Ademais, pode correr o risco de se transformarem em órgãos cartoriais que servem apenas para propagarem o que o executivo decide, em mecanismos de puro discurso governamental, sem que sejam implementadas ações como: reuniões, capacitações e acesso aos poderes locais. (OLIVEIRA; PEREIRA; OLIVEIRA, 2010).

\section{CONCLUSÃO}

O presente artigo teve por objetivo analisar os níveis de implementação dos instrumentos básicos de gestão municipal de assistência social nos municípios brasileiros, bem como, verificar quais os estados que se destacam na execução de tais instrumentos. As presentes contribuições apresentam algumas limitações, tendo em vista que a pesquisa analisou apenas um ano como corte temporal e não aprofundou aspectos qualitativos da gestão. No entanto, adquire importância ao avançar na análise da descentralização da assistência social em uma perspectiva diversa daquela que explora as competências dos entes federativos, direcionando a análise para a importância de discutir o compromisso municipal na implementação de inovações de gestão capazes de tornar as políticas sociais locais mais efetivas.

Nesse sentido o estudo mostrou que a assistência social no Brasil é reconhecida na constituição. A descentralização das ações, a ela imputadas, encontra-se devidamente regulamentada em legislação específica (LOAS), porém existem dificuldades no cumprimento 
das diretrizes propostas. Como exemplo, pode ser citada a inexistência de Lei municipal de regulamentação do SUAS na maioria dos municípios brasileiros.

De um modo geral, o nível de implementação de instrumentos de gestão municipal de assistência social é baixo, alcançando um percentual médio de $64 \%$ dos instrumentos avaliados. A análise do coeficiente de variação mostrou que não existe heterogeneidade significativa entre as unidades federativas. Contudo, foi possível identificar similaridades entre algumas delas. Os municípios dos estados do Mato Grosso do Sul, Paraná e o Distrito Federal lideraram na implementação dos mecanismos de gestão municipal de assistência social. No entanto, há ainda estados que apresentam certa fragilidade no aparato institucional para realizar uma boa gestão da área social, sendo o Rio Grande do Norte o caso com o menor nível de implementação.

Dentre os instrumentos avaliados, fica claro que a maior deficiência quanto ao emprego dos instrumentos disponíveis a uma melhor gestão municipal encontra-se na ausência de lei municipal de regulamentação do SUAS. Essa deficiência é preocupante e pode reduzir os impactos da política de assistência social dado que o SUAS é, atualmente, o responsável pela operacionalização das ações nessa área e a falta de regulamentação pode ser um risco ao direito do cidadão e a negação de uma assistência social assegurada por lei.

\section{REFERENCIAS}

ABRAMOVAY, R. Conselhos além dos limites. Estudos Avançados, São Paulo, v. 15, n. 43, p. 121-140, sept./dec. 2001.

ALMEIDA, C.; TATAGIBA, L. Os conselhos gestores sob o crivo da política: balanços e perspectivas. Serviço Social e Sociedade, São Paulo, v. 1, n. 109, p. 68-92, jan./mar. 2012.

ALVES, F. de A. A. Autonomia Municipal e interesse local como parâmetros a competência legislativa dos municípios. Revista da Faculdade de Direito de Campos, Rio de Janeiro, ano 4, n. 4, ano 5, n. 5, p. 527-581, 2003-2004.

BOVOLENTA, G. A. Os benefícios eventuais previstos na Loas: o que são e como estão. Serviço Social e Sociedade, São Paulo, n. 106, p. 365-387, 2011. 
INSTRUMENTOS DE GESTÃO DA ASSISTÊNCIA SOCIAL:

uma análise da realidade dos municípios brasileiros

BRASIL. Ministério do Desenvolvimento Social e Combate à Fome. Orientação aos municípios sobre regulamentação do Sistema Único de Assistência Social. Brasília, DF, 2012.

Planos de Assistência Social: diretrizes para

elaboração. 1. ed. Brasília, DF, 2008. (CapacitaSuas, v. 3). 2004.

Política Nacional de Assistência Social. Brasília, DF,

Secretaria Nacional de Assistência Social. Sistema

Único de Saúde. Política Nacional de Assistência Social PNAS/ 2004

- Norma Operacional Básica NOB/SUAS: construindo as bases para a implantação do Sistema Único da Assistência Social. Brasília, DF, 2005. Disponível em: $<$ http://www.assistenciasocial.al.gov.br/sala-deimprensa/arquivos/NOB-SUAS.pdf $>$. Acesso em: 2 nov. 2016.

Presidência da República. Constituição da República Federativa do Brasil. Brasília: DF, 1988. Disponível em: $<$ http://www. planalto.gov.br/ccivil_03/constituicao/constituicaocompilado.htm>. Acesso em: 13 jan. 2017.

Lei $\mathrm{n}^{\circ} 8.742$, de 7 de dezembro de 1993. Dispõe sobre a organização da Assistência Social e dá outras providências. Diário Oficial da União, Brasília, DF, 1993. Disponível em: $<$ http:// www.planalto.gov.br/ccivil_03/leis/18742.htm>. Acesso em: 30 out. 2016.

BULLA, L. C.; LEAL, M. L. M. A participação da sociedade civil no Conselho Municipal de Assistência Social: o desafio de uma representação democrática. Textos e Contextos, Porto Alegre, v. 3, n. 3, p.1-13, dez. 2004.

CAMPOS, E. C. S. Conselho Municipal de Assistência Social de Rolândia - CMAS: os desafios para a consolidação de uma gestão pública participativa. 2004. Dissertação (Mestrado em serviço social e Política Social) - Universidade Estadual de Londrina, 2004. Disponível em: <http://bdtd.ibict.br/vufind/Record/UEL_04efd414e46 484754d679952d6c2dd09>. Acesso em: 15 out. 2016.

CARRAZA, R. A. Curso de Direito Constitucional tributário. 3. ed. São Paulo: Revista dos Tribunais, 1991.

CARVALHO, A. C. de; LIMA, P. V. P. S.; SOUSA, R. P. A gestão municipal do saneamento básico no estado do Ceará. In: ENCONTRO REGIONAL DA SOBER, 8., 2013, Parnaíba. Anais... Parnaíba, 2013. 
Janaildo Soares de Sousa | Donária Linhares Minervino | Patrícia Verônica Pinheiro Sales Lima Leonardo Andrade Rocha $\mid$ Márcio Regys Rabelo de Oliveira

GARCIA, M. A negação da assistência social, a chegada da LOAS e a construção do SUAS no meio de vazios políticos, teóricos e metodológicos. Rio de Janeiro, 2011. Disponível em: $<$ www. marcelogarcia.com.br/negacaodaassistencia.pdf $>$. Acesso em: 13 jan. 2017.

HAIR JR., J. F. et al. Análise multivariada de dados. 5. ed. Porto Alegre: Bookman, 2005.

INSTITUTO BRASILEIRO DE GEOGRAFA E ESTATÍSTICA.

Perfil dos municípios brasileiros - Assistência Social 2013. Rio de Janeiro, 2013. Pesquisa de informações básicas municipais.

KLEBA, M. E; COMERLATTO, D.; FROZZA, K. M. Instrumentos e mecanismos de gestão: contribuições ao processo decisório em conselhos de políticas públicas. Revista de Administração Pública, Rio de Janeiro, v. 49, n. 4, p. 1059-1079, 2015.

LIMA, P. V. et al. Gestão municipal da segurança pública: responsabilidade dos municípios brasileiros no combate à violência. Revista Políticas Públicas, São Luís, v. 18, n. 2, p. 399-414, jul./dez. 2014.

MARQUES, D.; SANTOS, E. R. Sistema Único de Assistência Social (SUAS) e controle social: descentralização e participação política são a resposta? Sociedade em Debate, Pelotas, v. 22, n. 1, p. 44-70, 2016.

MELO, M. A. Crise federativa, guerra fiscal e hobbesianismo municipal: efeitos perversos da descentralização? In: OLIVEIRA, M. A. (Org.). Política e contemporaneidade no Brasil. Recife: Edições Bagaço, 1997.

NEVES, A. V.; SANTOS, C. de O. V.; SILVA, S. H. Conselhos Municipais de Assistência Social: novas competências para o trabalho do assistente social. Revista Katálysis, Florianópolis, v. 15, n. 2, p. 173-181, 2012.

OLIVEIRA, V. C. S.; PEREIRA, J. R.; OLIVEIRA, V. A. R. Os conselhos gestores municipais como instrumentos da democracia deliberativa no Brasil. Cadernos EBAPE.BR, Rio de Janeiro, v. 8, n. 3, p. $422-437$, set. 2010.

OLLAIK, L. G.; MEDEIROS, J. J. Instrumentos governamentais: reflexões para uma agenda de pesquisas sobre implementação de políticas públicas no Brasil. Revista de Administração Pública, Rio de Janeiro, v. 45, n. 6, p. 1943-1967, nov./dez. 2011. 
INSTRUMENTOS DE GESTÃO DA ASSISTÊNCIA SOCIAL:

uma análise da realidade dos municípios brasileiros

PERES, G. A. L; ALVES, A. L. C. O município e a gestão descentralizada e participativa da política de assistência social. Serviço Social e Realidade, Jardim Petraglia, v. 18, n. 1, p. 73-96. 2009.

QUINONERO, C. G. et al. Princípios e diretrizes da Assistência Social: da LOAS à NOB SUAS. O Social em questão, Rio de Janeiro, v. 17, n. 30, p. 47-69, 2013.

RONCONI, L. F. de A.; DEBETIR, E.; MATTIA, C. de. Conselhos gestores de políticas públicas: potenciais espaços para a coprodução dos serviços públicos. Contabilidade, Gestão e Governança, Brasília, DF, v. 14, n. 3, p. 46-59, set./dez. 2011.

SILVA, E. R. A. da. O direito à convivência familiar e comunitária: os abrigos para crianças e adolescentes no Brasil. Brasília, DF: IPEA/ CONANDA, 2004.

, J. A. da. Curso de Direito Constitucional Positivo. São Paulo: Malheiros, 2009.

, S. K. G. O município na Constituição Federal de 88. 1. ed. São Paulo: Juarez de Oliveira, 2003.

SOUSA, M. C; LIMA, P. V. P. S.; KHAN, A. S. Mecanismos de gestão municipal e a promoção dos direitos humanos. Revista de Administração Pública, Rio de Janeiro, v. 49, n. 4, p. 985-1009, 2015.

VAITSMAN, J.; ANDRADE, G. R. B. de; FARIAS, L. O. Proteção social no Brasil: o que mudou na assistência social após a Constituição de 1988. Ciência e Saúde Coletiva, Rio de Janeiro, v. 14, n. 3, p. 731741, 2009.

VÁZQUEZ, M. L. et al. Nível de informação da população e utilização dos mecanismos institucionais de participação social em saúde em dois municípios do Nordeste do Brasil. Ciência e Saúde Coletiva, Rio de Janeiro, v. 10, n. supl., p. 141-155, 2005.

WANDERLEY, M. C. A Assistência Social na Constituição Federal de 1988. Conteúdo Jurídico, Brasília, DF, 2015. Disponível em: $<$ http:// www.conteudojuridico.com.br/?artigos\&ver $=2.51978 \& \mathrm{seo}=1>$. Acesso em: 18 jan. 2017.

YAZBEK, M. C. Pobreza no Brasil contemporâneo e formas de seu enfrentamento. Serviço Social e Sociedade, São Paulo, n. 110, p. 288$322,2012$. 
Janaildo Soares de Sousa $\mid$ Donária Linhares Minervino $\mid$ Patrícia Verônica Pinheiro Sales Lima Leonardo Andrade Rocha $\mid$ Márcio Regys Rabelo de Oliveira

\section{NOTAS}

1 Segundo Silva (2009, p. 477) "Competência é faculdade juridicamente atribuída a uma entidade ou a um órgão ou agente do Poder Público para emitir decisões". No caso dos municípios, as decisões em questão são relativas aos interesses locais.

2 De acordo com o inciso I do art. 30 da CF/1988, "Compete aos Municípios: I - legislar sobre assuntos de interesse local". (BRASIL, 1988). A dificuldade na definição das competências do município está diretamente relacionada com à subjetividade do termo interesse local (ALVES, 2003-2004). Carraza (1991) o define como aquele que atende às necessidades do município, mesmo com alguma repercussão sobre as necessidades estaduais e nacionais. No entanto, não se tem clareza sobre o que separa o interesse local dos interesses dos estados e da união.

3 "Proteção social é definida como toda intervenção pública para ajudar indivíduos, domicílios e comunidades a administrar riscos ou apoiar os cronicamente pobres." (VAITSMAN; ANDRADE; FARIAS, 2009, p. 733).

4 O presente estudo adota a distribuição geográfica utilizada pelo IBGE.

5 Distribuição de municípios com IGMAS $=1$ por região: Centro Oeste $=7$, Nordeste $=16$, Norte $=2$, Sudeste $=5$ e Sul $=7$, o que equivale respectivamente a $1,5 \%, 0,9 \%, 0,4 \%, 0,3 \%$ e $0,6 \%$ do total de municípios de inseridos na região. 